\title{
Numerical Analysis of Wave Energy Extraction Performance According to the Body Shape and Scale of the Breakwater-integrated Sloped OWC
}

\author{
Hyunjai Yang ${ }^{\circledR 1}$, Eun-Hong Min ${ }^{\circledR 1}$ and WeonCheol Koo ${ }^{{ }^{2}}$ \\ ${ }^{1}$ Graduate Student, Department of NAOE, Inha University, Incheon, Korea \\ ${ }^{2}$ Professor, Department of NAOE, Inha University, Incheon, Korea
}

\begin{abstract}
KEY WORDS: Sloped oscillating water column, Wave energy converter, Numerical wave tank, Boundary element method, Wave energy extraction

ABSTRACT: Research on the development of marine renewable energy is actively in progress. Various studies are being conducted on the development of wave energy converters. In this study, a numerical analysis of wave-energy extraction performance was performed according to the body shape and scale of the sloped oscillating water column (OWC) wave energy converter (WEC), which can be connected with the breakwater. The sloped OWC WEC was modeled in the time domain using a two-dimensional fully nonlinear numerical wave tank. The nonlinear free surface condition in the chamber was derived to represent the pneumatic pressure owing to the wave column motion and viscous energy loss at the chamber entrance. The free surface elevations in the sloped chamber were calculated at various incident wave periods. For verification, the results were compared with the 1:20 scaled model test. The maximum wave energy extraction was estimated with a pneumatic damping coefficient. To calculate the energy extraction of the actual size WEC, OWC models approximately 20 times larger than the scale model were calculated, and the viscous damping coefficient according to each size was predicted and applied. It was verified that the energy, owing to the airflow in the chamber, increased as the incident wave period increased, and the maximum efficiency of energy extraction was approximately $40 \%$ of the incident wave energy. Under the given incident wave conditions, the maximum extractable wave power at a chamber length of $5 \mathrm{~m}$ and a skirt draft of $2 \mathrm{~m}$ was approximately $4.59 \mathrm{~kW} / \mathrm{m}$.
\end{abstract}

\section{Introduction}

Climate change has triggered the increase in the global temperature and subsequent sea level rise, causing significant damage, including drought, flood, and cold-weather damages. As part of multilateral cooperation and efforts to address the aforementioned problems, active international responses against climate change have materialized into the United Nations Framework Convention on Climate Change, Kyoto Protocol, and Paris Agreement. In line with the global trend, Korea has established a national target to reduce greenhouse gas (GHG) emissions by 2030, based on the principle of the nationally determined contribution (NDC) (Heo, 2016; Bae, 2021).

Accordingly, to reduce GHG emissions, there has been active research on new and renewable energy worldwide. Wave energy is an energy source with the highest energy density per unit area among marine energy sources, and is a highly promising resource that enables infinite sustainability with substantial potential. However, there are limitations to the utilization of wave energy: wave energy is subject to severe levels of seasonal and climatic variability, its various energy extraction methods and equipment are still under development, and a standardized power generation model for wave energy is yet to be established. The development of wave energy converters (WECs) has experienced steady advancements globally, and for some wave energy models, considerable progress has been made in commercialization, especially in the UK. Therefore, there is a significant demand for more active R\&D, including more research ideas and technical progress, as well as the commercialization and development of maintenance and operation methods.

The European Marine Energy Center Limited (EMEC), a worldleading marine energy research and development center in the UK, categorized WECs into oscillating water column (OWC), movable body type, and overtopping/terminator type WECs, according to the type of their primary energy conversion (EMEC, 2018). Among the various types of WEC, OWC is a method in which incident waves trigger the movement of the water surface inside the air chamber, which then causes the resulting surface elevation to generate airflow;

Received 3 May 2021, revised 14 June 2021, accepted 14 July 2021

Corresponding author Weoncheol Koo: +82-32-860-7348, wckoo@inha.ac.kr

(c) 2021, The Korean Society of Ocean Engineers

This is an open access article distributed under the terms of the creative commons attribution non-commercial license (http://creativecommons.org/licenses/by-nc/4.0) which permits unrestricted non-commercial use, distribution, and reproduction in any medium, provided the original work is properly cited. 
then, the generated airflow rotates the air turbine to extract energy. This generation method may be less efficient compared to the methods of converting wave energy into primarily usable energy; however, it has the advantage of easy maintenance because the air turbine for energy extraction is located above the water surface.

The OWC WEC can be divided into fixed and floating types, depending on the motion constraints of the structure. Various studies have been conducted on the fixed OWC WEC discussed in this study, and the findings of recent existing works have been reviewed in brief.

Koo et al. (2010) simulated the flow inside the OWC chamber of a general vertical chamber WEC using the 2D fully nonlinear numerical wave tank method. In addition, they analyzed the wave energy conversion efficiency in the time domain. Through the 2D wave tank experiment, the research was conducted with varying shape parameters of the chamber (ratios of the chamber and duct sizes) (Koo et al., 2012). Liu et al. (2010) compared the numerical results of 2D OWC WEC based on Desingularized boundary integral equation method (DBIEM) using the mixed Eulerian-Lagrangian method and the analytic results obtained by Sarmento and Falcao (1985). Subsequently, they analyzed the energy extraction efficiency according to the skirt draft of the structure. A number of studies have also been reported to analyze OWC WEC via the higher-order boundary element method (HOBEM), which considers the nonlinearity of each boundary element (Ning et al., 2015; Ning et al., 2020). Ning et al. (2016) compared the results of numerical analysis using HOBEM and the results obtained from water tank experiments. Kim et al. (2021) calculated the energy extraction efficiency of OWC WEC by applying the air damping coefficient in the chamber obtained by the theoretical solution, and compared their results with the results of previous studies with the application of the air damping coefficient.

In recent years, studies have been conducted on a sloped chamber WEC that can be installed via the connection to a breakwater built in a port or coast, by modifying a general vertical wall-type OWC. The sloped OWC has an energy extraction performance similar to that of the vertical OWC device, and its installation on the slope of the bottom-mounted breakwater, the existing onshore structure, is advantageous. As a recent study on the sloped OWC chamber, Park, et al. (2018b) measured the relative wave elevation inside the chamber, according to the change in skirt and width of a sloped OWC chamber, via a $2 \mathrm{D}$ wave tank experiment. In addition, by performing computational fluid dynamics (CFD) analysis, the wave elevation was calculated in two OWC chamber conditions: one with the chamber open, where the chamber pneumatic pressure is equal to the atmospheric pressure, and the other where the duct is installed above the chamber, such that chamber pneumatic pressure exists. The results obtained from the calculations were compared with those of the 2D water tank experiment. Via CFD analysis (using FLUENT), Gaspar et al. (2020) compared wave shapes inside vertical and $40^{\circ}$-sloped chambers. In the sloped chamber, run-up/down and sloshing were more dominantly observed than in the vertical chamber, and it was verified that the energy extraction efficiency of the sloped chamber was higher than that of the vertical chamber in terms of the wave period with the highest energy source.

The backward bent duct buoy (BBDB) type WEC is well-known as the floating OWC WEC. To examine the validity of the numerical modeling pneumatic damping coefficient, Kim et al. (2006) conducted a model test and measured the motion responses of BBDB in waves. In addition, the improvement in energy extraction efficiency was investigated by proposing a BBDB with rounded corners, which can reduce the viscous effect, via $2 \mathrm{D}$ wave tank experiments and numerical wave tank calculations (Kim et al., 2012; Lee et al., 2013; Kim et al., 2015).

In this study, a 2D fully nonlinear numerical wave tank method based on potential flow for a sloped-fixed OWC WEC was developed. Using the mixed Eulerian-Lagrangian method, numerical simulation was performed on the time domain for the nonlinear wave motion of the free surface inside and outside the sloped chamber. To consider the viscous effect in the analysis of potential flow based on non-viscous fluid, a viscous damping coefficient was added to the free surface boundary condition, and the flow change inside the chamber was calculated, considering the interactions with pneumatic pressure generated because of the free surface elevations in the OWC chamber, following the installation of a duct at the top of the chamber. The numerical results of this study were compared to the experimental results of previous studies (Park et al., 2018a, 2018b) to ensure reliability. The validated fully nonlinear numerical wave tank method can be calculated by selecting linear or nonlinear time domain analysis, and the proposed numerical method has the advantage of being relatively fast to obtain calculations, without having to conduct experiments for the investigation of wave steepness effect on the nonlinearity of the free surface. Using this study, each energy component of the sloped OWC WEC was calculated and compared with the incident wave energy, and it was verified that the energy of the entire WEC system was conserved. Finally, to elucidate the energy extraction and efficiency of the sloped OWC in the environment of real seas, the model size was increased by approximately 20 times, and the resulting changes in the viscous damping coefficient of the chamber were comparatively analyzed.

\section{Mathematical Formulation}

In this study, numerical modeling was performed for a sloped fixed OWC WEC, with the assumption that the fluid in the computational domain was an inviscid, incompressible, and irrotational potential fluid. The velocity potential representing the ideal fluid flow is defined as expressed in Eq. (1).

$$
V=\nabla \phi
$$

where $V$ and $\phi$ denote the velocity of the fluid particle and velocity potential, respectively. The velocity potential can easily represent a complex fluid flow phenomenon as a scalar value, and when the fluid 
flow satisfies the continuity equation and is an incompressible flow, the governing equation of the fluid domain can be represented using the Laplace equation:

$$
\nabla^{2} \phi=0
$$

The Laplace equation can be expressed as a boundary integral equation using Green's $2^{\text {nd }}$ identity, as expressed in Eq. (3).

$$
\alpha \phi_{i}=\iint_{\Omega}\left(G_{i j} \frac{\partial \phi_{j}}{\partial n}-\phi_{j} \frac{\partial G_{i j}}{\partial n}\right) d s
$$

where $\Omega$ denotes the fluid domain in which the calculation is performed. The solid angle $\alpha$ at the boundary is 0.5 , and the two-dimensional Green's function is $G_{i j}=-(1 / 2 \pi) \ln R_{1}$. Here, $R_{1}$ represents the distance between the source point and the field point at the boundary of the computation domain.

The free surface boundary condition is divided into two: kinematic and dynamic conditions, and it can be expressed as a nonlinear free surface boundary equation by taking the total derivative $(\delta / \delta t)$. In addition, under the assumption that the node points located on the free surface move in the same manner as the water particles (Material node approach, $\vec{\nu}=\nabla \phi$ ), they can be defined as expressed in Eqs. (4) and (5).

$$
\begin{aligned}
& \frac{\delta \eta}{\delta t}=\frac{\partial \phi}{\partial z} \\
& \frac{\delta \phi}{\delta t}=-g \eta+\frac{1}{2}|\nabla \phi|^{2}-\frac{P_{a}}{\rho}
\end{aligned}
$$

where $\eta, g, \rho$, and $P_{a}$ denote the free surface elevation, gravity, fluid density, and pneumatic pressure on the free surface, respectively. Outside the chamber, the pneumatic pressure is zero, based on atmospheric pressure, and when pneumatic pressure is present inside the chamber, its value changes in real time, according to the volume of the OWC chamber and the size of the nozzle (duct).

For the boundary condition of the incident wave, the propagating wave was generated by substituting the incident wave component into the boundary surface at the left end of the numerical wave tank, and the impermeable rigid boundary condition of Eq. (6) was applied to the boundary conditions of the tank bottom, right wall, and fixed structure. This indicates that the fluid velocity in the direction perpendicular to the boundary is zero.

$$
\frac{\partial \phi}{\partial n}=0
$$

When the incident wave enters inside the OWC chamber, energy loss occurs at the end of the chamber skirt owing to fluid viscosity. Considering this energy loss, in general, the equation for reduced pressure in the flow, which is proportional to the square of the fluid velocity, is expressed in Eq. (7).

$$
\triangle P=K_{L} \frac{1}{2} \rho \widetilde{V}^{2}
$$

where $K_{L}$ and $\widetilde{V}$ represent the loss coefficient and flow velocity, respectively. This equation can be expressed as Eq. (8), under the assumption that the water particle velocity $(\partial \phi / \partial n)$ is linearly proportional to the average vertical velocity of the water column $(\overline{\dot{\eta}})$. The viscous damping effect, which can occur when the incident wave enters the chamber, can be represented using the viscous damping coefficient $(\nu)$. By applying Eq. (8) to the dynamic free surface boundary condition in the chamber $(\delta \eta / \delta t=\partial \phi / \partial z)$, the energy reduction of the incident wave owing to fluid viscosity can be expressed as a viscous damping pressure term (Yang et al., 2019).

$$
P_{\nu}(t)=K_{L}^{*} \overline{\dot{\eta}}(t)=\nu \frac{\partial \phi(t)}{\partial n}
$$

where $K_{L}^{*}$ and $\overline{\dot{\eta}}$ represent the modified loss coefficient and average velocity of the surface elevation inside the OWC chamber, respectively. Inside the OWC chamber where the duct is installed, the pneumatic pressure is generated by the free surface elevation, and this is represented by assuming a linear relationship between the pneumatic pressure $\left(P_{a c}\right)$ in the OWC chamber and the velocity of the air flowing through the duct (Ning et al., 2015; Yang and Koo, 2020).

$$
P_{a c}(t)=C_{d m} U_{d}(t)
$$

where $C_{d m}$ denotes a linear pneumatic damping coefficient and $U_{d}(t)=U_{0} \sin \omega t$ represents a relationship between air flow rate with time. The air flow rate through the duct is determined by the ratios of the chamber and duct sizes. Therefore, the pneumatic pressure inside the chamber is defined as:

$$
P_{a c}(t)=\frac{C_{d m} \Delta V}{A_{d} \Delta t}
$$

where $A_{d}$ represents the area of the duct, $\Delta V / \Delta t$ is the rate of volume change inside the chamber, and the free surface boundary condition inside the OWC chamber, considering both the viscous damping effect and the pneumatic pressure effect (extractable by energy) owing to the duct, can be expressed by Eq. (11).

$$
\frac{\delta \phi}{\delta t}=-g \eta+\frac{1}{2}|\nabla \phi|^{2}-\frac{1}{\rho}\left(C_{d m} U_{d}+\nu \frac{\partial \phi}{\partial n}\right)
$$

The energy efficiency of the sloped OWC system was calculated using the boundary conditions described above; accordingly, the 
validity of the WEC numerical modeling and calculation results was verified. The incident wave energy flux can be expressed as the product of the incident wave energy and propagation velocity (group velocity). The fixed OWC WEC can be divided into four energy flux components: incident wave energy, reflected wave energy, maximum extractable energy of the turbine due to pneumatic pressure, and energy loss due to viscosity. Each of the four energy flux components can be expressed as follows (Koo et al., 2010; Kim et al., 2021).

$$
\begin{aligned}
& E_{i} C_{g}=\frac{1}{8} \rho g H_{i}^{2} C_{g} \\
& E_{R} C_{g}=\frac{1}{8} \rho g H_{R}^{2} C_{g} \\
& A P=\frac{1}{2} C_{d m} A_{d} U_{0}^{2} \\
& E L=\frac{1}{2} \nu \stackrel{\overline{B \eta}^{2}}{ }
\end{aligned}
$$

where $H_{i}$ and $H_{R}$ denote the incident and reflected wave heights, respectively, and $C_{g}$ represents the group velocity. The average energy extraction rate $(A P)$ is a value calculated per OWC chamber area and incident wave period, and it can be observed that the rate is proportional to the square of the air flow velocity in the nozzle (duct). The energy loss ( $E L$ ) owing to viscosity was also calculated similarly, and it was confirmed to be proportional to the square of the average vertical velocity $(\overline{\dot{\eta}})$ of the free surface in the chamber. Therefore, the sum of the reflected wave energy flux $\left(E_{R} C_{g}\right)$, average energy extraction $(A P)$, and energy loss $(E L)$ must be equal to the incident wave energy flux $\left(E_{i} C_{g}\right)\left(E_{R} C_{g}+A P+E L=E_{i} C_{g}\right)$. Using this equation, the validity of this numerical modeling was verified via the numerical analysis results, which indicate that the energy of the entire WEC system is conserved within the computation domain

\section{Numerical Modeling and Results}

The computational domain for the numerical modeling of the sloped chamber OWC WEC is illustrated in Fig. 1. Here, $d$ and $B$ denote the draft and thickness of the OWC chamber skirt, respectively, $L$ represents the width (gap) of the chamber, and $h$ represents the water depth. In addition, $B$ and $h$ are $0.09 \mathrm{~m}$ and $0.5 \mathrm{~m}$, respectively, and

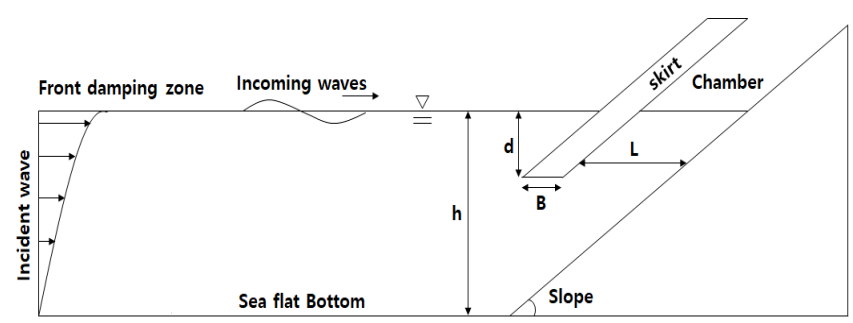

Fig. 1 Schematic diagram of a sloped OWC system with the open chamber condition these values were fixed in this study, and the slope angle of the skirt was set at $33.7^{\circ}$, which is similar to the angle of domestic sloped breakwaters, and would enable the installation of this model on a sloped breakwater in the future.

\subsection{Open Chamber}

To investigate the changes in the flow inside the chamber according to the OWC chamber conditions, a numerical model was applied under the condition that the pneumatic pressure inside the chamber is equal to atmospheric pressure, assuming the OWC chamber was open. For comparison with the experimental results of a previous study (Park et al., 2018b), the calculation was performed by applying the same incident wave conditions using a 1:20 scaled model. By setting the pneumatic pressure inside the chamber equal to atmospheric pressure, this model does not consider the power take-off (PTO). Tables 1 and 2 present the specifications various chamber dimensions and incident wave conditions used in the numerical analysis, respectively.

In the numerical analysis of this study, the viscosity of the fluid was not considered because the calculation was based on the assumption that the fluid was an inviscid, incompressible, and irrotational fluid. However, in real-sea conditions, energy attenuations occur, owing to the fluid viscosity between the structure and incident wave, as well as the energy loss according to the shape of the chamber structure. Therefore, to reflect the energy loss onto the numerical wave tank of the potential flow, the viscous damping coefficient should be substituted to consider the energy loss of the OWC system. For all cases in Table 1, arbitrary viscous damping coefficients were substituted to the numerical model, and the results closest to the experimental data (Park

Table 1 Specification of a numerical model of oscillating water column system $(B=0.09 \mathrm{~m}, h=0.5 \mathrm{~m})$

\begin{tabular}{ccc}
\hline Case & $d(\mathrm{~m})$ & $L(\mathrm{~m})$ \\
\hline 1 & 0.10 & 0.25 \\
2 & 0.10 & 0.20 \\
3 & 0.10 & 0.15 \\
4 & 0.15 & 0.20 \\
5 & 0.20 & 0.20 \\
\hline
\end{tabular}

Table 2 Incident wave conditions

\begin{tabular}{ccc}
\hline$T(\mathrm{~s})^{1)}$ & $H(\mathrm{~m})^{1)}$ & $H / \lambda^{1)}$ \\
\hline 1.00 & 0.066 & 0.044 \\
1.15 & 0.065 & 0.034 \\
1.30 & 0.057 & 0.025 \\
1.45 & 0.056 & 0.021 \\
1.60 & 0.055 & 0.018 \\
1.75 & 0.051 & 0.015 \\
1.90 & 0.045 & 0.012 \\
2.05 & 0.041 & 0.01 \\
\hline
\end{tabular}

1) $T=$ incident wave period, $H=$ incident wave height, $\lambda=$ wavelength 
et al., 2018b) with the same conditions were found, then, the adequate viscous damping coefficient was finally determined.

In Fig. 2, the results of the fully nonlinear time-domain calculations for surface elevation in the open chamber of Case 1 were compared under the conditions of incident wave period, wave height, and viscous coefficients at $1.75 \mathrm{~s}, 0.051 \mathrm{~m}$, and $\nu=0,0.5$, respectively. This corresponds to $\lambda / L=13.8$ in Fig. 3. The surface elevation is the value of the difference between the wave crest and wave trough divided by $1 / 2$. In this study, a ramp function twice the incident wave period $(T)$ was applied to generate stable incident waves in the fully nonlinear numerical wave tank. In addition, because OWC WEC is located at a distance approximately 4 times the incident wavelength from the wave maker of the numerical wave tank, it can be inferred that the surface elevation in the chamber occurs after approximately 4 periods $(t=4$ $T$ ). Under the above calculation conditions, in the calculation of the pure potential flow without viscous damping, it can be deduced that the elevation of the free surface inside the chamber is approximately 5 times higher than the incident wave, and when viscous damping effect is included, the elevation is approximately 3 times higher. Because the

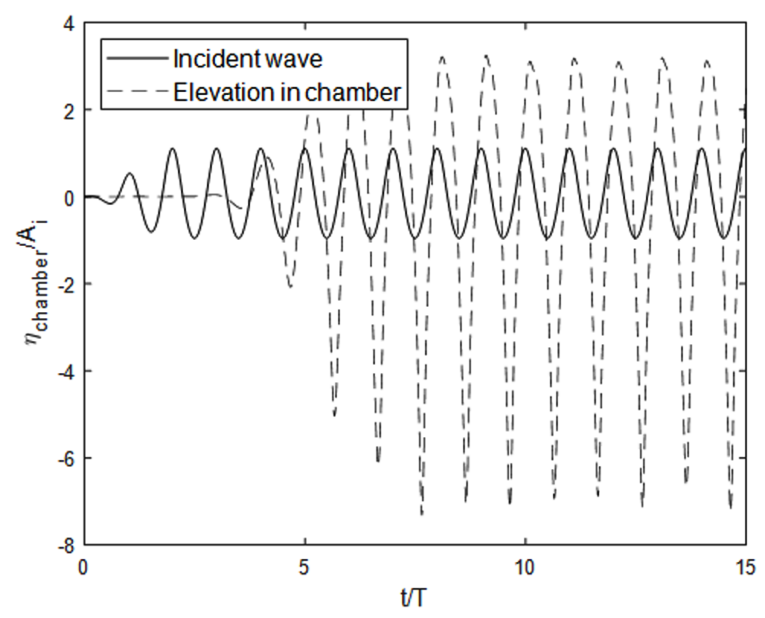

(a) $T=1.75 \mathrm{~s}, H=0.051 \mathrm{~m}, \nu=0$

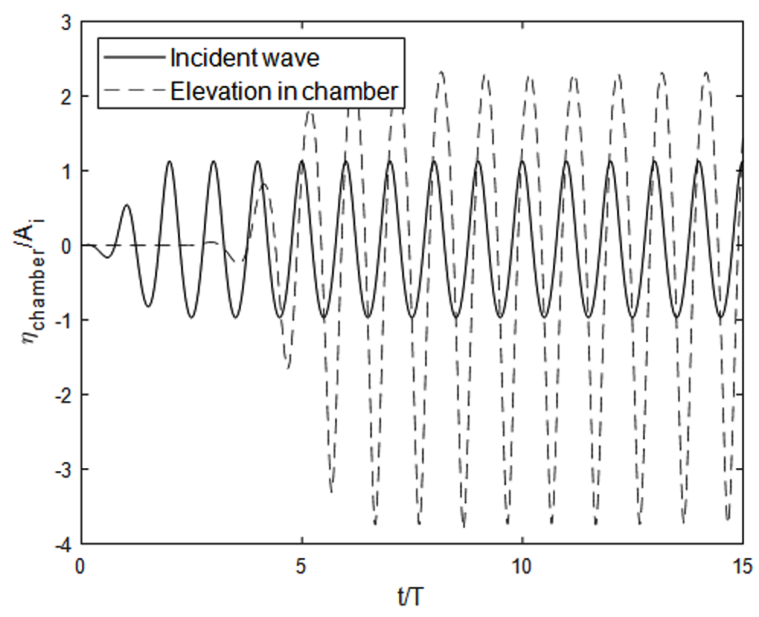

(b) $T=1.75 \mathrm{~s}, H=0.051 \mathrm{~m}, \nu=0.5$

Fig. 2 Time series of incident wave and chamber surface elevation in Case 1 (Open chamber)

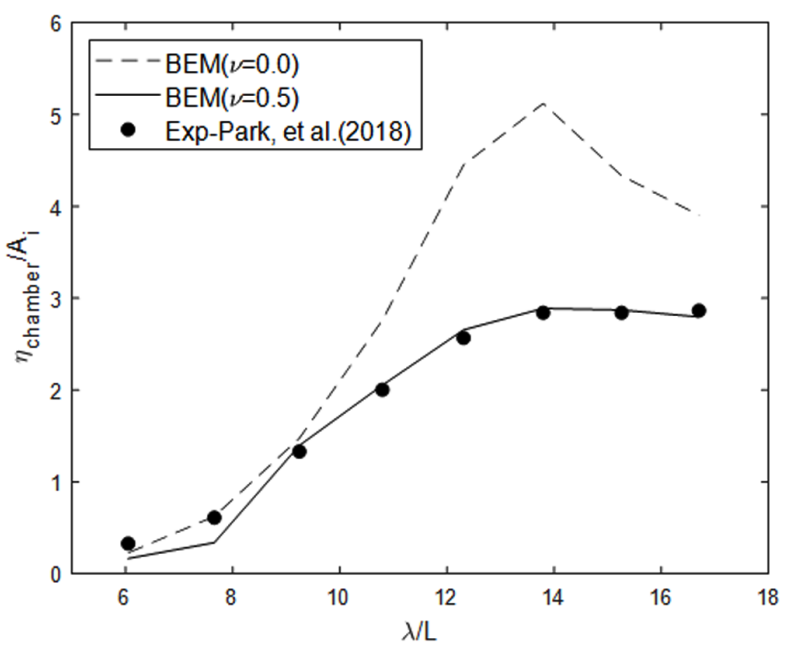

Fig. 3 Comparison of calculated surface elevations in the chamber with the experimental data at Case 1

surface elevation is high, as obtained from the results of the nonlinear calculation, the heights of the wave crest and wave trough are different, which triggers the asymmetry between the upper and lower surface elevations.

In Fig. 3, the calculated surface elevations in the chamber when viscous damping coefficient $(\nu=0.5)$ was applied to the numerical model, and those obtained by pure potential flow analysis $(\nu=0)$ are compared with the experimental data from Case 1. It can be observed that the results of the fully nonlinear numerical analysis with appropriate viscous damping coefficients agree well with the results of the $2 \mathrm{D}$ water tank experiment. In addition, when viscous damping was applied, the wave elevation in the chamber was reduced by approximately $28 \%$. In particular, it was verified that when the incident wavelength is longer than the chamber width (approximately 12-15 times), the energy attenuation due to viscosity increases. In the conditions of this study, it can be observed that the viscous damping effect was the largest when the incident wavelength was approximately 14 times the chamber width.

In Fig. 4, similar to the case in Fig. 3, the viscous damping coefficients, which can derive the numerical results closest to the experimental results, were obtained under the conditions of Cases 2-5, by comparing the results obtained from the numerical analysis and the 2D wave tank experiment (Park et al., 2018b). When $\nu=0.7$ in Case 2, $\nu=0.8$ in Case 3, $\nu=0.75$ in Case 4, and $\nu=0.8$ in Case 5 were applied to the numerical model, the results were closest to the experimental results. Therefore, the reliability of the OWC chamber numerical modeling (open chamber condition) was verified by calculating the appropriate viscous damping coefficient according to the chamber skirt draft and chamber width compared to the experimental values.

\subsection{Partial Open Chamber with Duct}

The total computation domain of the sloped fixed OWC WEC with a duct is illustrated in Fig. 5. 


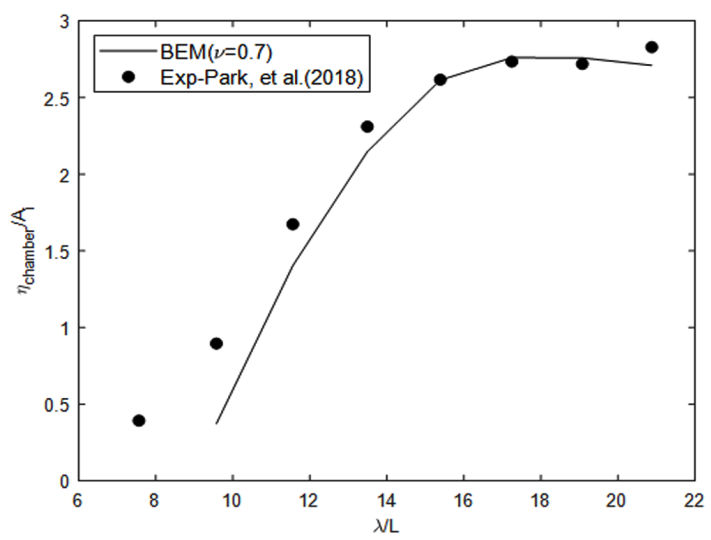

(a) Case $2(\nu=0.7)$

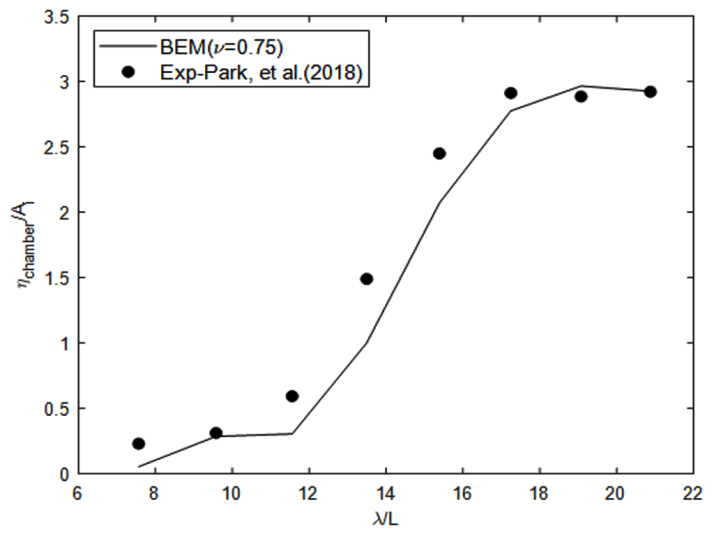

(c) Case $4(\nu=0.75)$

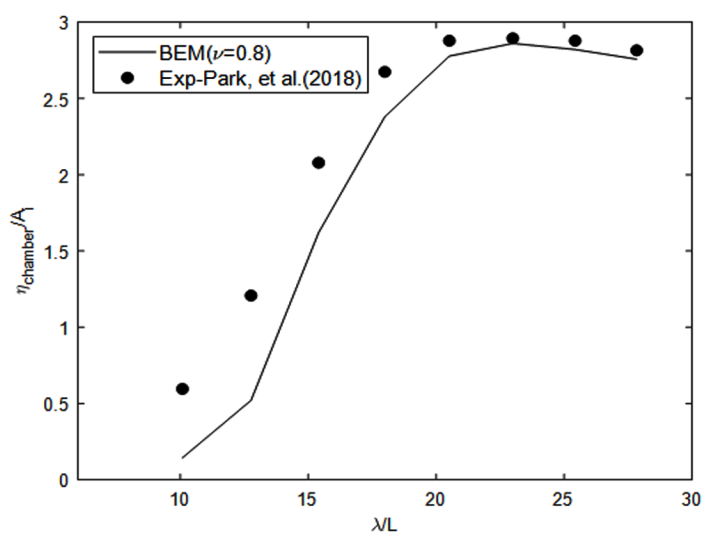

(b) Case $3(\nu=0.8)$

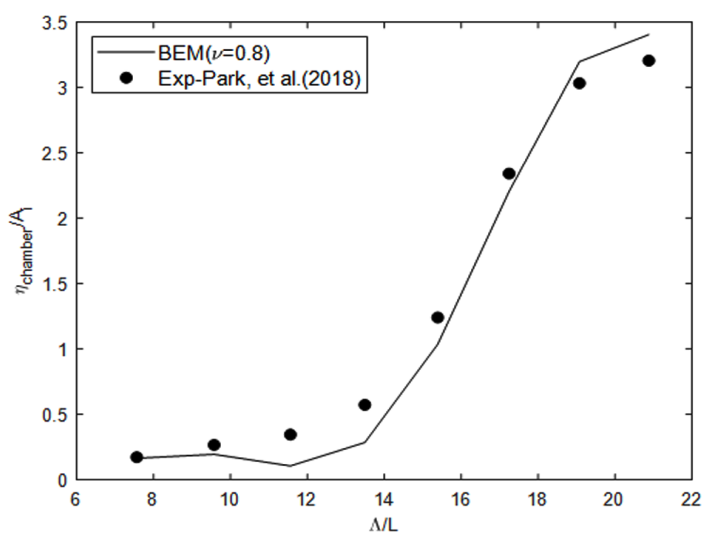

(d) Case $5(\nu=0.8)$

Fig. 4 Comparison of surface elevations in the chamber according to damping coefficients in various cases

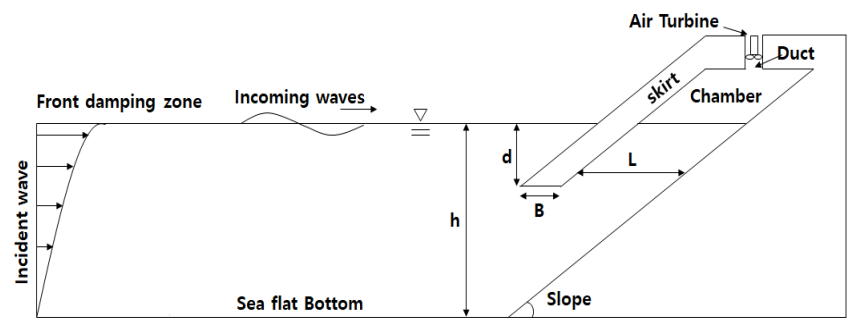

Fig. 5 Schematic of a sloped oscillating water column system with duct

Because the duct is installed in the upper part of the OWC chamber, pneumatic pressure occurs in the chamber. The pneumatic pressure inside the chamber is expressed by Eq. (10), which is substituted into the free surface boundary condition. The pneumatic damping coefficient $\left(C_{d m}\right)$ represents the maximum extractable wave energy that can be extracted from the air turbine installed in the duct, and it is related to the ratio of the chamber and duct sizes. The pneumatic damping coefficient value ( $C_{d m}=50$ ) was obtained by comparing the numerical results with the experimental results reported in Park et al. (2018a). The ratio of the chamber cross-sectional area to the duct area was approximately 120 . For the viscous damping coefficient, values obtained from a structure of the same size under the open chamber conditions were adopted.

Regarding Case $1(d=0.1 \mathrm{~m}, L=0.25 \mathrm{~m})$ conditions, with the incident wave period of $1.75 \mathrm{~s}$ and wave height of $0.051 \mathrm{~m}$, the incident wave and free surface elevation in the chamber were compared in time series (Fig. 6). Unlike the open chamber condition, the free surface elevation in the chamber was reduced to approximately $1 / 2$, compared to the incident wave height, and to approximately $1 / 4$ compared to the open chamber results, as the wave energy was extracted owing to the presence of the duct. To verify the pneumatic pressure effect, the results were compared with the results of the water tank experiment conducted by Park et al. (2018a), under the same conditions (Fig. 7). In

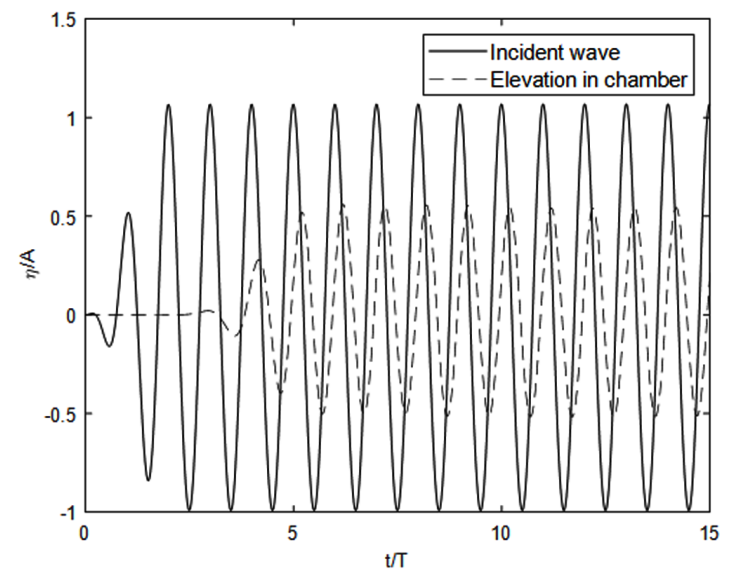

Fig. 6 Time series of incident wave and chamber elevation in Case $1(T=1.75 \mathrm{~s}, H=0.051 \mathrm{~m}, \nu=0.5$, Partial open chamber $)$ 
the open chamber $\left(C_{d m}=0\right)$ case, i.e., when the pneumatic pressure was zero, as the incident wavelength increased, the water surface in the chamber was elevated up to approximately 2.5 times the incident wave height; however, when a duct was installed in the chamber, the surface elevation decreased. This is because the pressure in the air chamber increased, owing to the installed duct; hence, free surface elevation in the chamber was suppressed. In fact, this can be regarded as the same effect when the wave energy was extracted with the air turbine of OWC, which led to the decrease in the energy in the chamber, and reduced the surface elevation. The above calculation is a case in which the air in the chamber is smoothly discharged from the duct without excessive compression, assuming that an air turbine with a large diameter, such as a Wells or an impulse turbine, is mainly used in the OWC. Therefore, in the case in which the viscous damping term has already been considered, the difference between the surface elevation in the open chamber and that in the chamber with the duct installed can be ascertained as the maximum extractable wave energy by the air turbine.

Fig. 8 presents the value of each energy flux component according to incident wave period and wave height conditions in Table 2 under Case $1(d=0.1 \mathrm{~m}, L=0.25 \mathrm{~m})$. It can be observed that the sum of the reflected wave energy flux, viscous damping energy, and air energy in

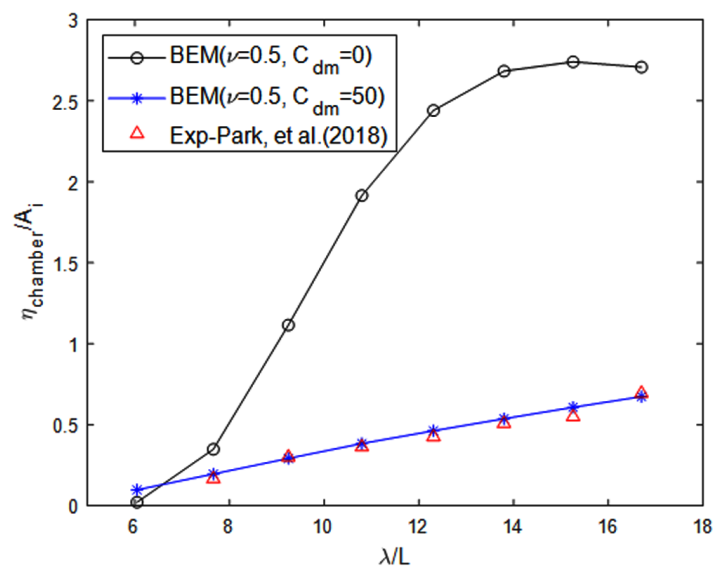

Fig. 7 Comparison of surface elevations in the chamber with a pneumatic damping coefficient

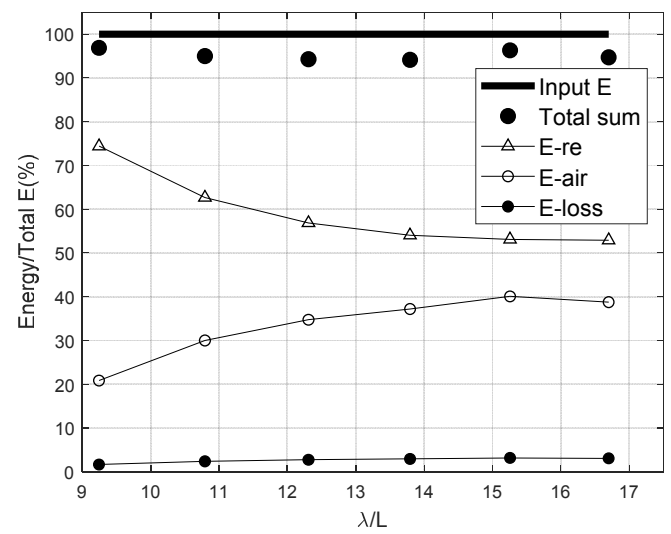

Fig. 8 Energy conservation of each energy flux component in Case $1(d=0.1 \mathrm{~m}, L=0.25 \mathrm{~m})$ the chamber is almost identical to the incident wave energy flux. This indicates that all energy flux components are accurately measured in the OWC WEC system. It can be observed that the energy (E-air) due to the air flow in the chamber increases as the incident wave period increases, which is approximately $20-40 \%$ of the incident wave energy. In addition, it can be observed that the reflected wave energy decreases with the increase in the period, which is at least $50 \%$ or more of the incident wave energy. In the future, it is necessary to develop a method that minimizes the reflected wave energy, such that more wave energy can be extracted. No significant change exists in the viscous damping energy relative to the change in the incident wave period.

\subsection{Real Scale Model}

In this study, numerical analysis was performed on a 1:20 scaled model compared to the real size model for comparative validation with existing experimental results (Park et al., 2018a, 2018b), and via this comparison, it was determined that the validity of the numerical model was verified. Based on this finding, an appropriate viscous damping coefficient was calculated while increasing the size of the numerical model to predict the energy extraction (power) of the sloped OWC WEC on a real scale.

Fig. 9 illustrates the comparison with experimental values by substituting appropriate viscous damping coefficients for the real scale model ( $L=5 \mathrm{~m}, d=2 \mathrm{~m}$ ), which is enlarged by 20 times compared to the scale model ( $L=0.25 \mathrm{~m}, d=0.1 \mathrm{~m})$ under Case $1(L / d=2.5)$. Accordingly, the changes in the viscous damping coefficient $\left(\nu / \nu_{0}, \nu_{0}\right.$ is the viscous coefficient of the scale model $(d=0.1 \mathrm{~m})$ can be illustrated in Fig. 10 for various scale factors $(2,5,10,15,20$ times the initial scale model) of Cases 2, 4, and 5, with the same chamber width, and Cases 1-3 with the same draft of the chamber skirt. The obtained results confirmed that the viscous damping coefficient increased with a similar trend, according to the scale in all Cases.

To investigate the energy efficiency in actual sea environments, a calculation was performed under the wave conditions shown in Table 3 , considering the average wave period of the Korean coast. The

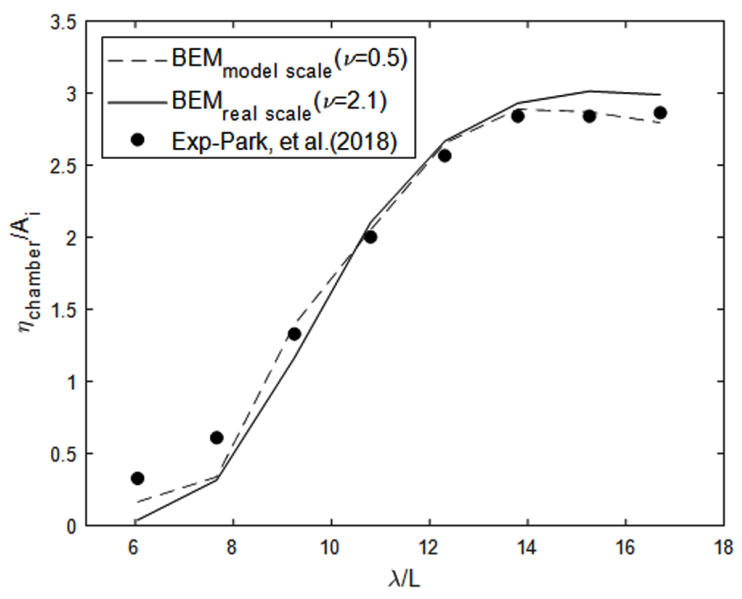

Fig. 9 Comparison of surface elevation in the chamber at model and real scales (Model: $d=0.1 \mathrm{~m}, L=0.25 \mathrm{~m}, \nu=0.5$, Real: $d=2 \mathrm{~m}, L=5 \mathrm{~m}, \nu=2.1$ ) 


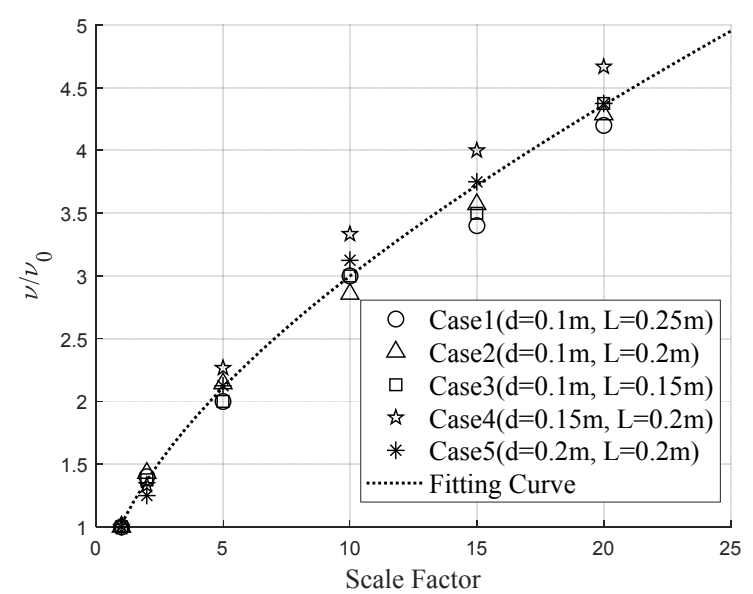

Fig. 10 Viscous damping coefficients with scale factor

viscous damping coefficient of the real scale model (20-times larger model, scale factor $=20$ ) was obtained for each case. The maximum extractable energy was obtained by applying the calculated damping coefficient to the numerical analysis, and the obtained results are presented in Fig. 11. Because the pneumatic damping coefficient $\left(C_{d m}\right)$ is related to the volume ratio, $C_{d m}=50$ used in the scale model was adopted without alterations. When comparing the maximum extractable energy under the given incident wave conditions, the obtained value was the largest in Case 1. Table 3 presents the maximum extractable energy under the incident wave conditions, as

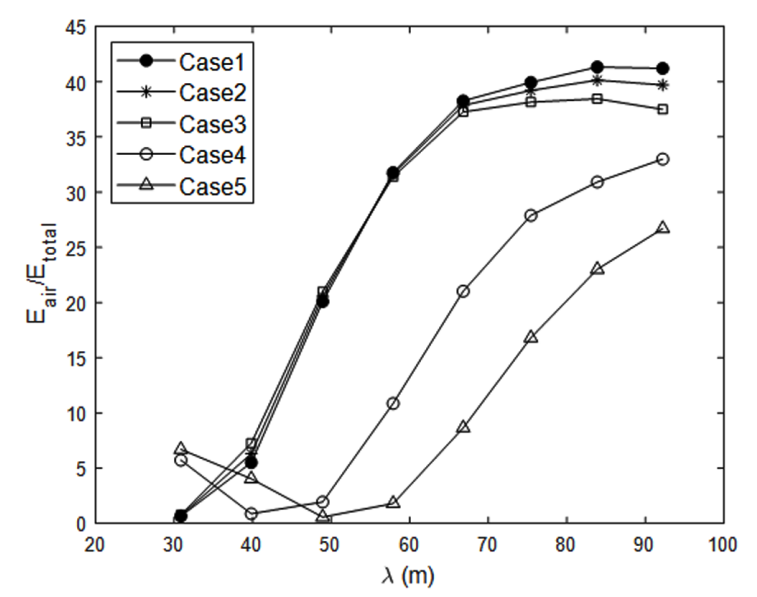

Fig. 11 Comparison of E-air for Cases $1-5$

Table 3 Real scale condition and E-air at Case $1(L=5 \mathrm{~m}, d=2 \mathrm{~m})$

\begin{tabular}{cccc}
\hline$T$ _real $(\mathrm{s})$ & $H_{-}$real $(\mathrm{m})$ & $\lambda(\mathrm{m})$ & E-air $(\mathrm{kW} / \mathrm{m})$ \\
\hline 4.47 & 1.32 & 30.85 & 0.05 \\
5.14 & 1.3 & 39.81 & 0.55 \\
5.81 & 1.14 & 48.90 & 1.94 \\
6.48 & 1.12 & 57.87 & 3.48 \\
7.16 & 1.1 & 66.80 & 4.57 \\
7.83 & 1.02 & 75.41 & 4.59 \\
8.50 & 0.9 & 83.87 & 4.01 \\
9.17 & 0.82 & 92.20 & 3.53 \\
\hline
\end{tabular}

well as the shape of Case 1 for the conditions. Under the conditions of period ( $T=7.83 \mathrm{~s})$, wavelength $(\lambda=75.41 \mathrm{~m})$, and wave height ( $H=$ $1.02 \mathrm{~m}$ ), the value of the maximum extractable energy was up to 4.59 $\mathrm{kW} / \mathrm{m}$, which is approximately $40 \%$ of the incident wave energy.

\section{Conclusion}

In this study, a sloped fixed OWC WEC was modeled using a 2D fully nonlinear numerical wave tank method based on potential flow. The viscous damping coefficients were calculated according to the chamber dimension, by comparing the numerical results obtained under the condition of an open OWC chamber with experimental results. Based on the obtained results, the wave elevation in the chamber was calculated relative to the chamber width and skirt draft. To consider the numerical model in which the pneumatic pressure is generated by the free surface elevations owing to the application of the duct in the OWC chamber, the pneumatic damping coefficient representing the maximum extractable energy from the air turbine installed in the duct. The viscous damping coefficient obtained from the open chamber condition were substituted to calculate the wave elevation in the chamber, which was compared to that of the experimental results under the same conditions, to verify the validity of the calculation results. In addition, energy conservation was verified in the system by calculating all energy flux components of the WEC system. The obtained results indicated that approximately $40 \%$ of the incident wave energy can be extracted under the conditions of Case 1. This value includes the energy loss due to turbine efficiency and viscous damping. Although the reflected wave energy gradually decreased in the long wave condition, the results indicated that the value was at least $50 \%$ or more of the incident wave energy. Therefore, a method for minimizing the reflected wave energy is required to extract the energy in further studies. In addition, a regular wave was adopted as an incident wave to accurately determine the energy extraction relationship, according to the shape of the OWC structure. The change in the viscous damping coefficient of the structure was predicted according to the scale factor, by expanding the numerical analysis scale model by approximately 20 times. By implementing the changes to the numerical analysis, the maximum extractable wave energy (approximately $4.59 \mathrm{~kW} / \mathrm{m}$ ) in the real scale structure (approximately 20 times that of the Case 1 model) was calculated, which was approximately $40 \%$ of the incident wave energy. In the future, for the accurate estimation of energy extraction in actual marine environmental conditions, follow-up studies with the applications of irregular wave conditions are required, and further studies on mutual verification should be conducted via a CFD simulation analysis based on viscous fluid.

\section{Funding}

This research was supported by the Basic Research Project of Science and Engineering, National Research Foundation of Korea (NRF-2018R1D1A1B07040677). 


\section{References}

Bae, S. (2021). 2050 Carbon Neutral. Electric Power, 1, 70-71. European Marine Energy Centre Ltd. (EMEC). (2018). Marine Energy/Wave Devices. Retrieved 20 April 2021 from http://www. emec.org.uk/marine-energy/wave-devices/

Gaspar, L.A., Teixeira, P.R.F., \& Didier, E. (2020). Numerical Analysis of the Performance of Two Onshore Oscillating Water Column Wave Energy Converters at Different Chamber Wall Slopes. Ocean Engineering, 201, 107119. https://doi.org/ 10.1016/j.oceaneng.2020.107119

Heo, S. (2016). Climate Change and Concerted Actions by Mankind. Journal of Korean Social Trend and Perspective, 96, 214-220.

Kim, D.M., Min, E.H., \& Koo, W. (2021). Numerical Study on the Optimal Shape and Performance of an Oscillating Water Column Using Analytic Air Damping Coefficients and Numerical Wave Tank. Journal of The Korean Society for Marine Environment \& Energy, 24(1), 1-8. https://doi.org/10.7846/JKOSMEE.2021. 24.1.1

Kim, J.H., Lew, J.M., Hong, D.C., \& Hong, S.W. (2006). A Study on Motion and Wave Drift Force of a BBDB Type OWC Wave Energy Device. Journal of Ocean Engineering and Technology, 20(2), 22-28.

Kim, S.J., Koo, W., \& Kim, M.H. (2015). Nonlinear Time-domain NWT Simulations for Two Types of a Backward Bent Duct Buoy (BBDB) Compared with 2D Wave-tank Experiments. Ocean Engineering, 108(1), 584-593. https://doi.org/10.1016/j.oceaneng. 2015.08.038

Kim, S.J., Kwon, J., Kim, J.D., Koo, W., Shin, S., \& Kim, K. (2012). Experimental Study of Hydrodynamic Perfromance of Backward Bent Duct Buoy (BBDB) Floating Wave Energy Converter. Journal of Ocean Engineering and Technology, 26(6), 53-58. https://doi.org/10.5574/KSOE.2012.26.6.053

Koo, W.C., Kim, M.H., \& Choi, Y.R. (2010). Numerical Analysis of Chamber Flow and Wave Energy Conversion Efficiency of a Bottom-Mounted Oscillating Water Column Wave Power Device. Journal of the Society of Naval Architects of Korea, 47(3), 388-397. https://doi.org/10.3744/SNAK.2010.47.3.388

Koo, W., Kwon, J.S., Kim, J.D., Kim, S.J., Kim, M.W., \& Choi, M.K. (2012). Experimental Study of Shape Parameter of Land-based OWC Wave Energy Converter. Journal of Ocean Engineering amd Technology, 26(3), 33-38. https://doi.org/10.5574/KSOE. 2012.26.3.033

Lee, K.R., Koo, W., \& Kim, M.H. (2013). Fully Nonlinear Time-domain Simulation of a Backward Bent Duct Buoy Floating Wave Energy Converter Using an Acceleration Potential Method. International Journal of Naval Architecture and Ocean Engineering, 5(4), 513-528. https://doi.org/10.2478/ IJNAOE-2013-0150

Liu, C., Huang, Z.H., Keung, A.L.W., \& Geng, N. (2010). A
Numerical Study of Wave Energy Converter in the Form of an Oscillating Water Column Based on a Mixed EulerianLagrangian Formulation. Proceedings of the ASME 2010 29th International Conference on Ocean, Offshore and Arctic Engineering, Shanghai, China, 589-596. https://doi.org/10.1115/ OMAE2010-21056

Ning, D.Z., Guo, B.M., Wang, R.Q., Vyzikas, T., \& Greaves, D. (2020). Geometrical Investigation of a U-shaped Oscillating Water Column Wave Energy Device. Applied Ocean Research, 97, 102105. https://doi.org/10.1016/j.apor.2020.102105

Ning, D.Z., Shi, J., Zou, Q.P., \& Teng, B. (2015). Investigation of Hydrodynamic Performance of an OWC (Oscillating Water Column) Wave Energy Device Using a Fully Nonlinear HOBEM (Higher-Order Boundary Element Method). Energy, 83, 177188. https://doi.org/10.1016/j.energy.2015.02.012

Ning, D.Z., Wang, R.Q., Zou, Q.P., \& Teng, B. (2016). An Experimental Investigation of Hydrodynamics of a Fixed OWC Wave Energy Converter. Applied Energy, 168, 636-648. https://doi.org/10.1016/j.apenergy.2016.01.107

Park, S., Kim, K.H., Nam, B.W., Kim, J.S., \& Hong, K. (2018a). A Study on the Performance Evaluation of the OWC WEC Applicable to Breakwaters using CFD. Journal of The Korean Society for Marine Environment \& Energy, 21(4), 317-327. https://doi.org/10.7846/JKOSMEE.2018.21.4.317

Park, S., Nam, B.W., Kim, K.H., \& Hong, K. (2018b). Parametric Study on Oscillating Water Column Wave Energy Converter Applicable to Breakwater. Journal of Advanced Research in Ocean Engineering, 4(2), 66-77. https://doi.org/10.5574/JAROE. 2018.4.2.066

Sarmento, A.J.N.A., \& Falcao, A.F. (1985). Wave Generation by an Oscillating Surface-pressure and Its Application in Wave-energy Extraction. Journal of Fluid Mechanics, 150, 467-485. https://doi.org/10.1017/S0022112085000234

Yang, H.J., Koo, W., \& Kim, S.J. (2019). A Study on th Shape Parameters of a Sloped Oscillating Water Column WEC Using a 2D Full Nonlinear Numerical Wave Tank. Proceedings of 2019 Fall Conference of the Society of Naval Architects of Korea, Gyeongju, Korea, 390-393.

Yang, H.J., \& Koo, W.C. (2020). A Study on the Performance Evaluation of the Sloped Oscillating Water Column WEC Using a 2D Full Nonlinear Numerical Wave Tank. Proceedings of 2020 Fall Conference of the Korean Society of Ocean Engineers, Korea, 224-227.

\section{Author ORCIDs}

$\begin{array}{ll}\text { Author name } & \text { ORCID } \\ \text { Yang, Hyunjai } & 0000-0001-6258-2315 \\ \text { Min, Eun-Hong } & 0000-0003-1045-9475 \\ \text { Koo, WeonCheol } & 0000-0002-4384-0996\end{array}$

